\title{
A New Task Scheduling Algorithm for Maximizing the Distributed Systems Efficiency
}

\author{
Amal El-Nattat \\ Computer Science \& Eng. Dept., \\ Faculty of Electronic Eng. \\ Menouf 32952, Egypt.
}

\author{
Nirmeen A. El-Bahnasawy \\ Computer Science \& Eng. Dept., \\ Faculty of Electronic Eng. \\ Menouf 32952, Egypt.
}

\author{
Ayman El-Sayed \\ Computer Science \& Eng. Dept., \\ Faculty of Electronic Eng. \\ Menouf 32952, Egypt.
}

\begin{abstract}
Efficient task scheduling is essential to obtain high performance in distributed computing environment. Achieving a better makespan is a key issue in designing and development of task scheduling algorithms. Several algorithms have been proposed for homogeneous and heterogeneous distributed computing systems. In this paper, we proposed a new static scheduling algorithm called Leveled DAG Prioritized Task (LDPT) to efficiently schedule tasks on homogeneous distributed computing systems. LDPT aims to improve the efficiency of the system by minimizing the schedule length.
\end{abstract}

\section{Keywords}

Task scheduling; Homogeneous distributed computing systems; Precedence constrained parallel applications; Directed Acyclic Graph.

\section{INTRODUCTION}

Distributed systems have emerged as powerful platforms for executing parallel applications. They are efficient systems that are known to solve tasks and problems in a feasible and fast way. A distributed system can be defined as a computing system in which services are provided by a pool of loosely coupled computers collaborating over a network working for a common goal [1]. It can be homogeneous (in which processors are identical in capabilities and functionality) or heterogeneous (in which processors are different).

In distributed computing environment, an application is usually decomposed into several independent and/or interdependent sets of cooperating tasks. These tasks are represented by a Directed Acyclic Graph (DAG). DAG is a graph consists of a set of vertices or nodes and a set of edges $G(V, E)$ in which each node represents a task and each edge represents a communication between two tasks (the two tasks are dependent on each other). The weight associated with each node represents the computation cost of the task and the weight associated with each edge represents the communication cost between two tasks. If two dependent tasks are executed on the same processor, then the communication cost between them is considered to equal zero. Figure 1 shows an example of a simple task graph (DAG). In the figure, $\mathrm{t} 1$ is called predecessor (or parent) of $\mathrm{t} 2$ and $\mathrm{t} 2$ is called successor (or child) of $\mathrm{t} 1$. The edge between $\mathrm{t} 1$ and $\mathrm{t} 2$ means that $\mathrm{t} 2$ can start execution only after $\mathrm{t} 1$ finishes its execution.

The efficient scheduling of application tasks is critical to achieve high performance in parallel and distributed systems. The objective function of scheduling is to map the tasks onto the processors and order their execution so that task precedence requirements are satisfied and minimum schedule length (or makespan) is obtained [2].Task-scheduling algorithms are broadly classified into two classes: static and dynamic. When the characteristics of an application, such as execution time of tasks and data dependencies between tasks are known in advance, the scheduling algorithm is known as static model.
Static task scheduling takes place during compile time before running the distributed application. Whereas in the dynamic scheduling decisions are made at run time [3].

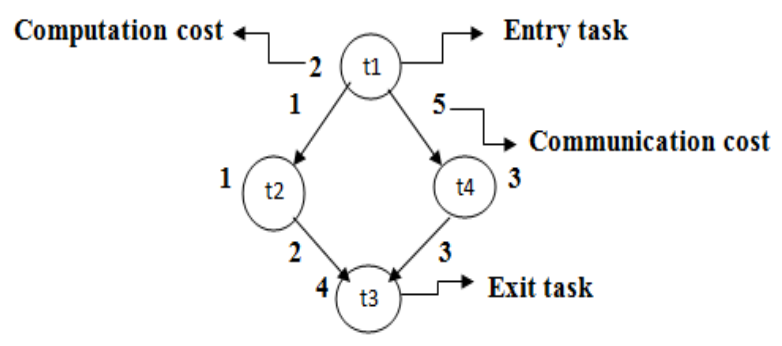

Fig 1: Example of a DAG

Over the past few decades, research efforts are mainly focused on the problem of task scheduling on algorithms running on homogenous and heterogeneous systems mainly with the objective of reducing the overall execution time of the tasks. Topcuoglu et al. [4] have presented HEFT and CPOP scheduling algorithms for heterogonous processors. Luiz et al. [5] have developed lookahead-HEFT algorithm, which look ahead in the schedule to make scheduling decisions. Eswari, R. and Nickolas, S. [6] have proposed PHTS algorithm to efficiently schedule tasks on the heterogeneous distributed computing systems. Rajak and Ranjit [7] have presented a queue based scheduling algorithm called TSB to schedule tasks on homogeneous parallel multiprocessor system. Ahmed, S.G.; Munir, E.U.; and Nisar, W. [8] have developed genetic algorithm called PEGA that provide low time complexity than standard genetic algorithm (SGA). Xiaoyong Tang; Kenli Li; Renfa Li; and Guiping Liao [9] have presented a listscheduling algorithm called HEFD for heterogeneous computing systems. Nasri, W. and Nafti, W. [10] have developed a new DAG scheduling algorithm for heterogeneous systems that provide better performance than some well-known existing scheduling algorithms.

In homogeneous environment, the researchers have explored many heuristic task-scheduling algorithms such as ISH [11], MCP [12], ETF [13], DLS [14], MH [15], and B-level [16]. Among these algorithms, B-level provides the best performance in terms of schedule length, speedup, and efficiency. As a result, B-level is used for scheduling tasks in EASy (Energy Aware Scheduling) algorithm [17]. LDPT provides better performance than B-level algorithm. We expect when applying LDPT instead of B-level in EASy algorithm, it will provide better results and leads to lower power consuming.

In this paper, the problem of scheduling precedence constrained parallel tasks on homogeneous physical machines (PMs) is addressed. We proposed a new static scheduling algorithm (LDPT). The goal of LDPT is to improve the performance of the system than B-level algorithm [16]. 
The remainder of this paper is organized as follows. Section 2 provides an overview of the related work algorithm. The proposed algorithm is discussed in section 3. Section 4 presents performance evaluation results of the proposed algorithm. Section 5 introduces a discussion of energy awareness. Finally, conclusion is reviewed in section 6 .

\section{RELATED WORK ALGORITHM}

B-level algorithm is a list based scheduling algorithm. It depends on sorting all tasks in the graph into a list and then schedules them one by one. Before the scheduling begins, the b-level values of all tasks in a task graph are computed and sorted into a scheduling list in decreasing order of their b-level values. Then, tasks are picked from the sorted list and assigned to PMs one by one. Each task is assigned to the processor which minimize the earliest start time of that task. If $\mathrm{LPath}_{\mathrm{i}}=$ $\left\{n_{i}, n_{i+1}, \ldots, n_{\text {exit }}\right\}$ is the longest path from node $n_{i}$ to exit node so the b-level value for the task $n_{i}$ is defined as:

b-leveli $=\Sigma$ njE node(LPathi) $w j+\Sigma e j \in e d g e(L P a t h i)$ cj ---- (1)

\section{OUR PROPOSED SCHEDULING ALGORITHM (LDPT)}

LDPT is a list based scheduling algorithm. It depends on dividing the DAG into levels with considering the dependency conditions among tasks in the DAG. The algorithm has two phases: (1) Task prioritization phase, (2) Processor selection phase.

\subsection{Task Prioritization Phase:}

In this phase, the DAG is divided into levels then, the tasks in each level will be sorted into a list based on their computation cost in decreasing order. The ties are broken by the communication cost between the task and all of its childs in the next level.

The priority for each task is $\mathrm{W}_{\mathrm{j}}\left(\mathrm{n}_{\mathrm{i}}\right)$ for tasks that have non equal computation cost values or $\mathrm{Cj}\left[(\mathrm{ni}) \mathrm{j}, \sum_{x=1}^{N}\left(n_{x}\right) \mathrm{j}+1\right]$ for tasks having equal computation cost values, Where $\mathrm{W}_{\mathrm{j}}\left(\mathrm{n}_{\mathrm{i}}\right)$ is the computation cost of the specified task $\left(n_{i}\right)$ in the $j$ level where $1 \leq \mathrm{j} \leq \mathrm{T}, \mathrm{T}$ is the total number of levels and $1 \leq \mathrm{i} \leq \mathrm{N}, \mathrm{N}$ is the total number of tasks. $\mathrm{C}_{\mathrm{j}}\left[\left(\mathrm{n}_{\mathrm{i}}\right)_{\mathrm{j}}, \sum_{x=1}^{N}\left(n_{x}\right)_{\mathrm{j}+1}\right]$ determines the sum of communication costs between task $\left(n_{i}\right)$ in $j$ level and all of its children in $\mathrm{j}+1$ level. A child task is denoted by $\left(n_{x}\right)$ where $1 \leq \mathrm{x} \leq \mathrm{N}$.

\subsection{Processor Selection Phase:}

In this phase, the tasks are picked from the list one by one and assigned to the processor that will minimize the earliest start time of the task, with taking into consideration the insertionbased policy. The insertion policy means that if there is an idle time slot on the processor between two already scheduled tasks and it was enough for executing the task, then the task is assigned on that processor in this idle slot without violating precedence constraints. In other words, a task can be scheduled earlier if there is a period of time between two tasks already scheduled on processor $(\mathrm{P})$, where $\mathrm{P}$ runs idle. If two processors provide the same start time for the task then, the task is assigned to the processor on which most of its parents are scheduled. The Earliest Start Time of a task $n_{i}$ on a processor $P_{j}$ is defined as:

$\operatorname{EST}\left(n_{x}, P_{m}\right)=\max \left[\operatorname{TAvailable}\left(P_{m}\right), \max \left\{\operatorname{AFT}\left(n_{i}\right)+c_{x, i}\right\}\right]--(2)$

Where TAvailable $\left(P_{m}\right)$ is the earliest time at which processor $P_{m}$ is ready. $\operatorname{AFT}\left(n_{i}\right)$ is the Actual Finish Time of a task $n_{i}$ (the parent of task $\mathrm{nx}$ ) on the processor $P_{m} . \quad c_{n, i}$ is the communication cost from task $n_{i}$ to task $n_{x}, c_{k, i}$ equal zero if the predecessor task $t_{k}$ is assigned to processor $P_{m}$. For the entry task, $\operatorname{EST}\left(n_{\text {entry }}, P_{m}\right)=0$. Figure (2) shows the pseudo code of LDPT algorithm.

Generate the DAG
Divide the DAG into levels according to their
communicated dependency
Sort the constructed levels according to dependency
ordering
Sort tasks according to [their computation costs then their
direct communication of its next level] in descending order
While there are unscheduled levels do
While there are unscheduled tasks do
\[ \text { For each level do } \]
Find the task with the highest computation cost
If there are tasks have equal computation cost
Then
End for each
End while
Find the processor that minimizes the Earliest
Start Time of the selected task
Assign the task to the selected processor
Remove the selected task from the list

Fig 2: .Leveled DAG Prioritized Task (LDPT) algorithm.

\subsection{Case Study}

Consider the DAG shown in figure (3), assume the system has two processors ( $\mathrm{P} 0, \mathrm{P} 1)$. Table 1 shows the computation cost for each task; table 2 shows the b-level value for each task. Both b-level, LDPT algorithms generate a list of tasks that shows the execution order of them. For b-level algorithm, the tasks are sorted in the list in decreasing order according to their b-level value that is computed by using equation 1 . For LDPT algorithm, the DAG is divided into levels and the tasks are sorted in each level in descending order according to their computation cost. Table 3 shows the lists generated by b-level and LDPT algorithms. Figure (4.a, 4.b) shows the gantchart generated by B-level and LDPT algorithms respectively. Both algorithms assign the selected task to the processor that minimizes the start time (EST) of it. For example, in figure 4.a, the EST for task $t_{6}$ on $p_{0}$ is 2 and the EST for t6 on $p_{1}$ is 1 , so the task t6 is scheduled on p1. The data ready time (DRT) for task $t_{5}$ on $p_{0}$ is 4 and the DRT for $t_{5}$ on $p_{1}$ is 6 , so the task is scheduled on $\mathrm{p}_{0}$. In figure 4.b, the same manner if followed but with taking into consideration the insertion based policy. For example, in figure 4.b, the EST for task $t_{7}$ on $p_{0}$ is 15 and the EST for $t_{7}$ on $p_{1}$ is 18 while the DRT for $t_{7}$ on $p_{0}$ is 13 and the DRT for $t_{7}$ on $p_{1}$ is 9 . It is shown that $p_{1}$ is idle from 9 to 14 and this period is enough for executing $t_{7}$, so $t_{7}$ is inserted in this idle time period (between $t_{2}$ and $t_{8}$ ) on $p_{1}$. In addition, task $\mathrm{t}_{10}$ is inserted by the same manner. From figure 4, it is shown 
that the schedule length (the finish time of the last task scheduled from the DAG) resulted from B-level and LDPT algorithms is 26,24 unit of time respectively.

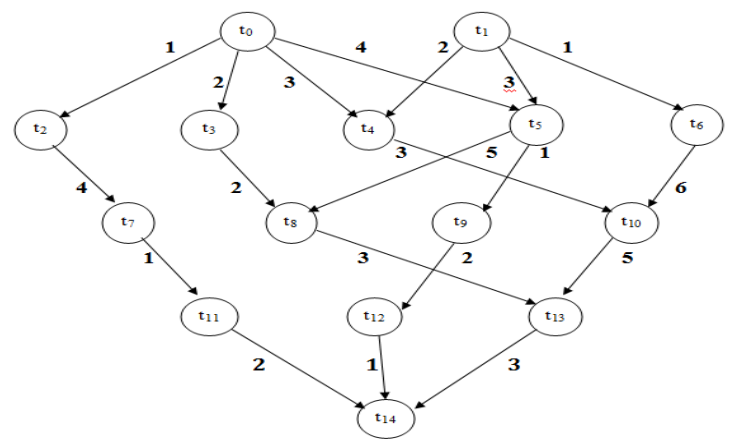

Fig 3: Sample DAG

Table1. Computation cost

\begin{tabular}{|c|c|}
\hline Task & Computation Cost \\
\hline $\mathbf{t}_{\mathbf{0}}$ & $\mathbf{2}$ \\
\hline $\mathbf{t}_{1}$ & $\mathbf{1}$ \\
\hline $\mathbf{t}_{2}$ & $\mathbf{4}$ \\
\hline $\mathbf{t}_{3}$ & $\mathbf{2}$ \\
\hline $\mathbf{t}_{4}$ & $\mathbf{3}$ \\
\hline $\mathbf{t}_{5}$ & 3 \\
\hline $\mathbf{t}_{6}$ & 4 \\
\hline $\mathbf{t}_{7}$ & 6 \\
\hline $\mathbf{t}_{8}$ & 2 \\
\hline $\mathbf{t}_{9}$ & 2 \\
\hline $\mathbf{t}_{10}$ & $\mathbf{5}$ \\
\hline $\mathbf{t}_{11}$ & $\mathbf{1}$ \\
\hline $\mathbf{t}_{12}$ & 3 \\
\hline $\mathbf{t}_{13}$ & \\
\hline $\mathbf{t}_{14}$ & \\
\hline
\end{tabular}

Table 2.The computed b-level values for tasks in sample DAG

\begin{tabular}{|c|c|}
\hline Task & b-leve \\
\hline $\mathbf{t}_{\mathbf{0}}$ & 28 \\
\hline $\mathbf{t}_{\mathbf{1}}$ & 27 \\
\hline $\mathbf{t}_{\mathbf{2}}$ & 16 \\
\hline $\mathbf{t}_{\mathbf{3}}$ & 20 \\
\hline $\mathbf{t}_{\mathbf{4}}$ & 19 \\
\hline $\mathbf{t}_{5}$ & 22 \\
\hline $\mathbf{t}_{\mathbf{6}}$ & 25 \\
\hline $\mathbf{t}_{7}$ & 11 \\
\hline $\mathbf{t}_{\mathbf{8}}$ & 14 \\
\hline
\end{tabular}

\begin{tabular}{|c|c|}
\hline $\mathbf{t}_{9}$ & 17 \\
\hline $\mathbf{t}_{10}$ & 14 \\
\hline $\mathbf{t}_{11}$ & 7 \\
\hline $\mathbf{t}_{12}$ & 9 \\
\hline $\mathbf{t}_{13}$ & 7 \\
\hline $\mathbf{t}_{14}$ & $\mathbf{3}$ \\
\hline
\end{tabular}

Figure 4 depicts the gantchart generated by B-level and LDPT algorithms. From the figure, it is shown that the schedule length generated from B-level algorithm is 26 unit time while the schedule length generated from LDPT algorithm is 24 unit time. In case of B-level, we observe that there is some periods in which processors are idle, while in case of LDPT, there is no idle periods on the processors. According to this result, the overall running time of the application will be decreased and the efficiency of the system will be improved.

Table 3.Task lists for b-level and LDPT algorithms

\begin{tabular}{|c|c|c|}
\hline Execution & b-level & LDPT \\
\hline 1 & $t_{0}$ & $t_{0}$ \\
\hline 2 & $t_{1}$ & $t_{1}$ \\
\hline 3 & $t_{6}$ & $t_{6}$ \\
\hline 4 & $t_{5}$ & $t_{3}$ \\
\hline 5 & $t_{3}$ & $t_{5}$ \\
\hline 6 & $t_{4}$ & $t_{4}$ \\
\hline 7 & $t_{9}$ & $t_{2}$ \\
\hline 8 & $t_{2}$ & $t_{9}$ \\
\hline 9 & $t_{8}$ & $t_{8}$ \\
\hline 10 & $t_{10}$ & $t_{7}$ \\
\hline 11 & $t_{7}$ & $t_{10}$ \\
\hline 12 & $t_{12}$ & $t_{12}$ \\
\hline 13 & $t_{13}$ & $t_{11}$ \\
\hline 14 & $t_{11}$ & $t_{13}$ \\
\hline 15 & $t_{14}$ & $t_{14}$ \\
\hline
\end{tabular}

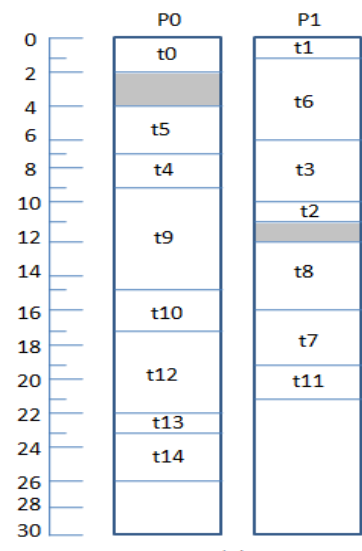

(a)

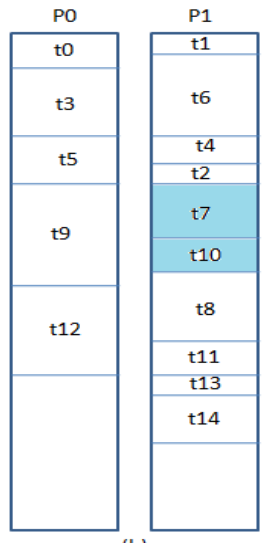

(b)
Fig 4: The schedules generated by (a) B-level algorithm (b)LDPT algorithm for sample DAG 


\section{RESULTS AND PERFORMANCE EVALUATION \\ 4.1 Simulation Environment}

To evaluate the performance of our developed LDPT algorithm, a simulator had been built using visual C\# .NET 4.0 on machine with: Intel(R) Core(TM) i3 CPU M 350 @ $2.27 \mathrm{GHz}, \mathrm{RAM}$ of $4.00 \mathrm{~GB}$, and the operating system is window 7, 64-bit.

To test the performance of B-level and LDPT algorithms, a set of randomly generated graphs is created by varying a set of parameters that determines the characteristics of the generated DAGs. These parameters are described as follows:

DAG size ( $n$ : the number of tasks in DAG). Density (d: the probability of existence edge between $n_{i}$ in level $l_{j}$ and $n_{x}$ in the next level level ${ }_{j+1}$ for DAG. Where, $i, x=1,2, \ldots, N$, and $N$ is the number of tasks, $\mathrm{j}=1,2, \ldots, \mathrm{T}$, and $\mathrm{T}$ is the number of levels in DAG). With six different numbers of processors varying from $2,4,8,16,32$ and 64 processors. For each number of processors, six different DAG sizes have been used varying from $10,20,40,60,80$ and 100 nodes.

\subsection{Evaluation Metrics}

Schedule length, speed up, and efficiency are the most important metrics used for evaluating performance of scheduling algorithms. In addition, energy consumption is defined as a metric since we are focused on minimizing of the energy consumed by the PMs and NDs. Schedule length is the maximum finish time of the last task (exit task) scheduled from the DAG.

\section{Schedule length $=\operatorname{Max}(A F T($ nexit $)$}

Where AFT(nexit) is the actual finish time of the exit task.Speed up is defined as the ratio of the schedule length generated from executing the application on one processor to the schedule length generated from executing the application on multiple parallel processors.

Speed $u p=\frac{\underset{\operatorname{Min}}{\operatorname{MicP}\left[\sum_{\text {nieV }} w(i, j)\right]}}{S L}$ (4)

Where $w(i, j)$ means the weight of task ni on processor $\mathrm{pj}$ and SL means the schedule length. Efficiency is the inverse of speed up.

Efficiency $=\frac{1}{\text { speedup }}$

\subsection{Experimental Results}

The schedule length generated by b-level and LDPT algorithms is shown in figure $5,6,7,8,9,10$ for $10,20,40,60,80,100$ tasks respectively and the results are recorded in table 4 . According to the results, the schedule length is decreased that will minimize the running time of the application. The improvement ratio in schedule length is $(1 \%)$. Figure 11, 12, $13,14,15,16$ show a comparative study of the speed up of blevel and LDPT algorithms in case of 2, 4, 8, 16, 32, 64 processors respectively. Table 5 shows the speedup results of $\mathrm{B}$-level and LDPT algorithms. From the results, we can see that the improving ratio in speed up is $(1.58 \%)$. From figure 17,18 , $19,20,21,22$ we can see that LDPT is more efficient than blevel. The improvement ratio in efficiency is $(1.38 \%)$. Table 6 shows the efficiency results of B-level and LDPT algorithms.

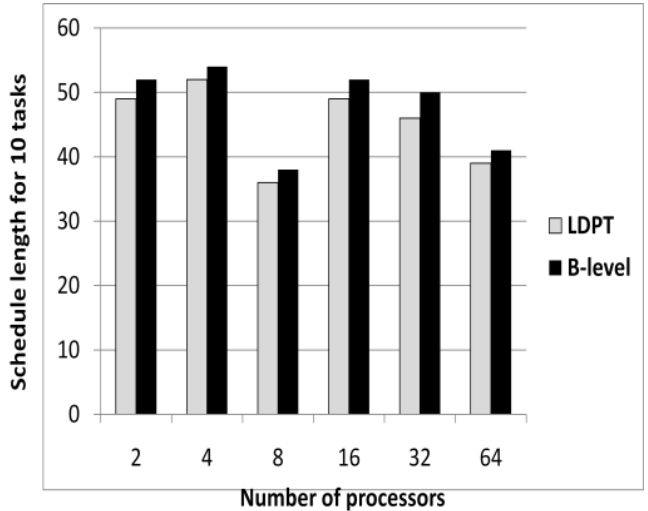

Fig 5: Schedule length for 10 tasks

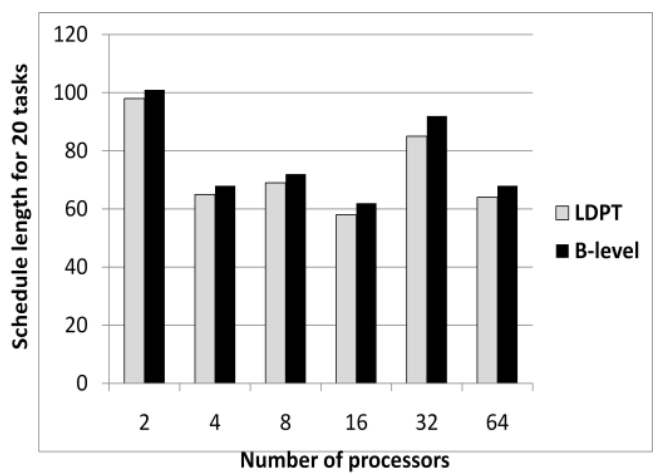

Fig 6: Schedule length for 20 tasks

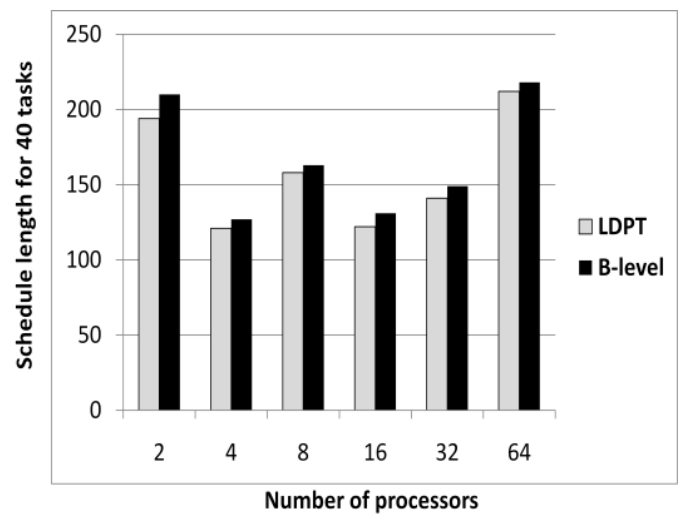

Fig 7: Schedule length for 40 task

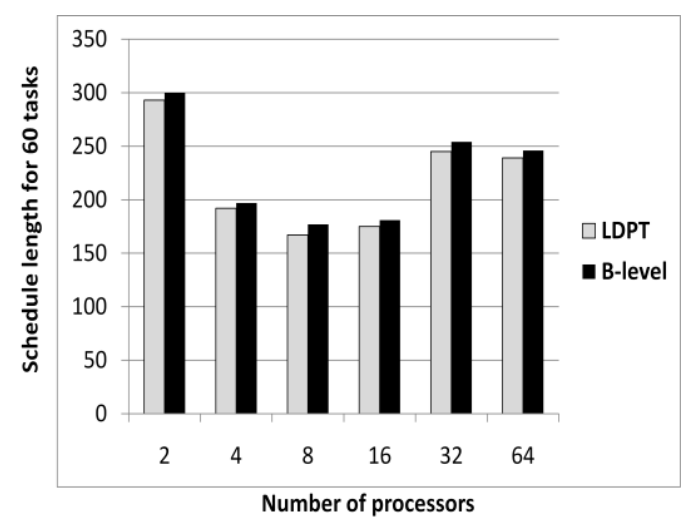

Fig 8: Schedule length for 60 task 
Figure $5,6,7,8,9,10$ depict the schedule length versus number of tasks with varying number of processors $2,4,8,16$, and 32 processors. It is shown that the schedule length in case of applying LDPT algorithm is less than B-level algorithm. This is because B-level algorithm depends on paths idea and this will increase the communication overhead during assigning tasks on processors. On the other side, LDPT algorithm depends on levels idea that will minimize the communication overhead during assigning tasks on processors. Another reason is that B-level algorithm must calculate the blevel value for each task before scheduling so that, the arithmetic calculation in LDPT is less than B-level algorithm which leads to minimize the complexity factor.

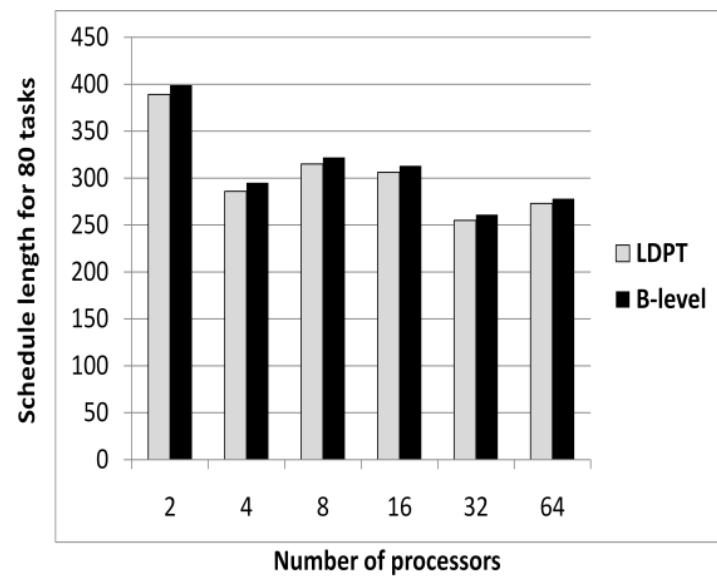

Fig 9: Schedule length for 80 task

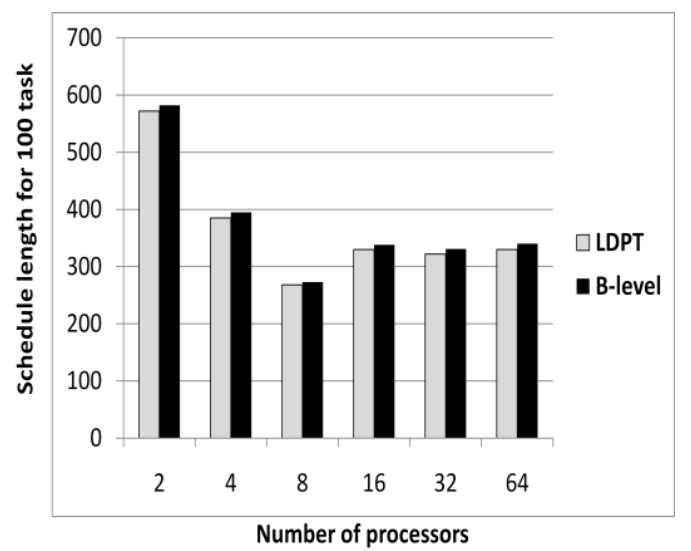

Fig 10: Schedule length for 100 task

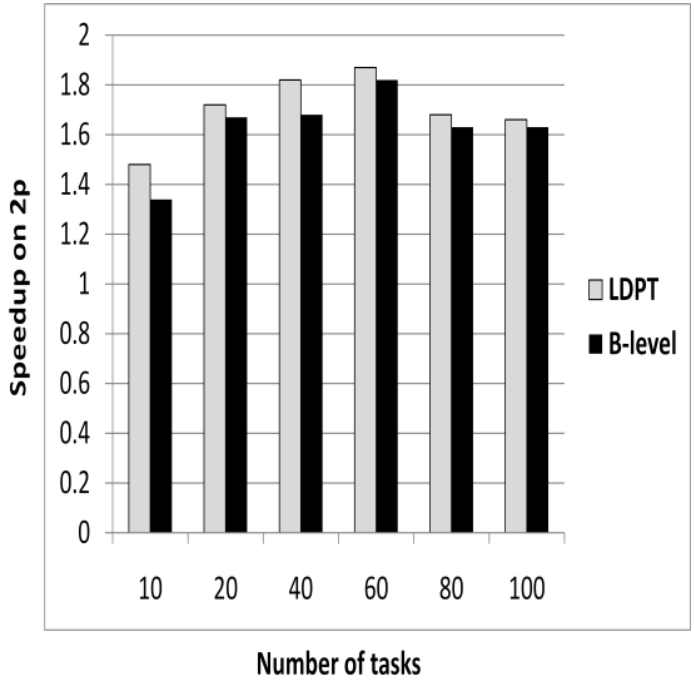

Fig 11: Speedup on 2 processors

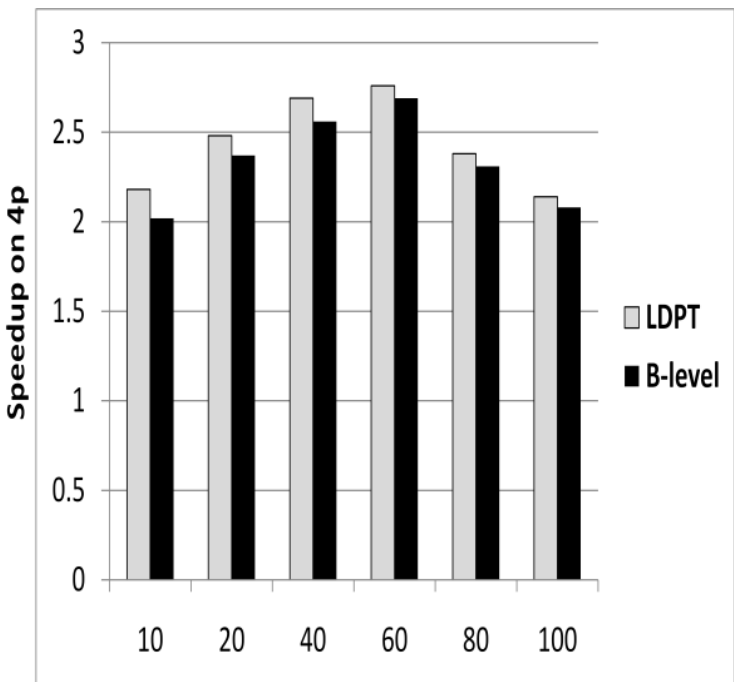

Number of tasks

Fig 12: Speedup on 4 processors

Table 4. Schedule length resulted from B-level and LDPT algorithms

\begin{tabular}{|c|c|c|c|c|c|c|c|c|c|c|c|c|}
\hline \multirow{2}{*}{$\begin{array}{l}\text { Number } \\
\text { of tasks }\end{array}$} & \multicolumn{2}{|c|}{2 processor } & \multicolumn{2}{|c|}{4 processor } & \multicolumn{2}{|c|}{8 processor } & \multicolumn{2}{|c|}{16 processor } & \multicolumn{2}{|c|}{32 processor } & \multicolumn{2}{|c|}{64 processor } \\
\hline & $\begin{array}{c}\text { B- } \\
\text { level }\end{array}$ & LDPT & $\begin{array}{c}\text { B- } \\
\text { level }\end{array}$ & LDPT & $\begin{array}{c}\text { B- } \\
\text { level }\end{array}$ & LDPT & $\begin{array}{c}\text { B- } \\
\text { level }\end{array}$ & LDPT & $\begin{array}{c}B \text { - } \\
\text { level }\end{array}$ & LDPT & $\begin{array}{c}B \text { - } \\
\text { level }\end{array}$ & LDPT \\
\hline 10 & 52 & 49 & 54 & 52 & 38 & 36 & 52 & 49 & 50 & 46 & 41 & 39 \\
\hline 20 & 101 & 98 & 68 & 65 & 72 & 69 & 62 & 58 & 92 & 85 & 68 & 64 \\
\hline 40 & 210 & 194 & 127 & 121 & 163 & 158 & 131 & 122 & 149 & 141 & 218 & 212 \\
\hline 60 & 300 & 293 & 197 & 192 & 177 & 167 & 181 & 175 & 254 & 245 & 246 & 239 \\
\hline 80 & 399 & 389 & 295 & 286 & 322 & 315 & 313 & 306 & 261 & 255 & 278 & 273 \\
\hline 100 & 582 & 572 & 395 & 385 & 273 & 268 & 338 & 330 & 331 & 322 & 340 & 330 \\
\hline
\end{tabular}


Table 5. Speedup resulted from B-level and LDPT algorithms

\begin{tabular}{|c|c|c|c|c|c|c|c|c|c|c|c|c|}
\hline \multirow{2}{*}{$\begin{array}{l}\text { Number of } \\
\text { processors }\end{array}$} & \multicolumn{2}{|c|}{10 tasks } & \multicolumn{2}{|c|}{20 tasks } & \multicolumn{2}{|c|}{40 tasks } & \multicolumn{2}{|c|}{60 tasks } & \multicolumn{2}{|c|}{80 tasks } & \multicolumn{2}{|c|}{100 tasks } \\
\hline & $\begin{array}{c}B- \\
\text { level }\end{array}$ & LDPT & $\begin{array}{c}\text { B- } \\
\text { level }\end{array}$ & LDPT & $\begin{array}{c}\text { B- } \\
\text { level }\end{array}$ & LDPT & $\begin{array}{c}\text { B- } \\
\text { level }\end{array}$ & LDPT & $\begin{array}{c}B- \\
\text { level }\end{array}$ & LDPT & $\begin{array}{c}\text { B- } \\
\text { level }\end{array}$ & LDPT \\
\hline 2 & 1.34 & 1.48 & 1.67 & 1.72 & 1.68 & 1.82 & 1.82 & 1.87 & 1.63 & 1.68 & 1.63 & 1.66 \\
\hline 4 & 2.02 & 2.18 & 2.37 & 2.48 & 2.56 & 2.69 & 2.69 & 2.76 & 2.31 & 2.38 & 2.08 & 2.14 \\
\hline 8 & 2.51 & 2.64 & 2.15 & 2.25 & 2.01 & 2.08 & 2.77 & 2.93 & 2.17 & 2.22 & 3.19 & 3.25 \\
\hline 16 & 1.86 & 1.98 & 2.65 & 2.83 & 2.53 & 2.72 & 2.83 & 2.93 & 2.16 & 2.21 & 2.55 & 2.61 \\
\hline 32 & 1.65 & 1.8 & 2 & 2.16 & 2.31 & 2.44 & 2.12 & 2.2 & 2.6 & 2.66 & 2.52 & 2.59 \\
\hline 64 & 1.39 & 1.52 & 2.26 & 2.41 & 1.59 & 1.64 & 2.11 & 2.18 & 2.53 & 2.57 & 2.34 & 2.42 \\
\hline
\end{tabular}

Figure 11, 12, 13, 14, 15, 16 depict speedup versus number of processors with varying number of tasks $(20,40,60,80,100)$. It is shown that LDPT algorithm provide better speed up than B-level algorithm. This is because in case of LDPT algorithm, all processors have finished the execution of tasks earlier than B-level algorithm.

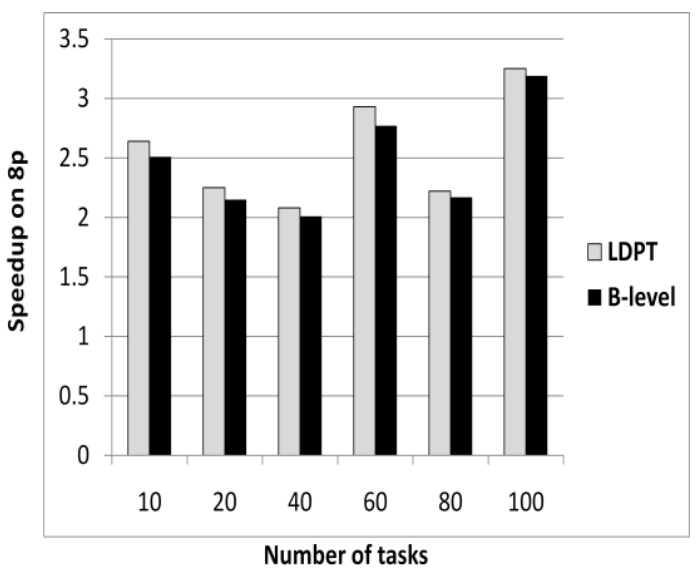

Fig 13: Speedup on 8 processors

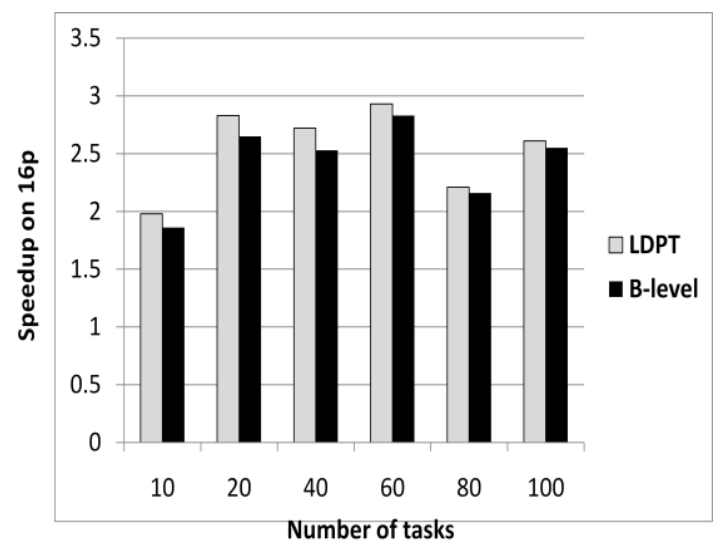

Fig 14: Speedup on 16 processors

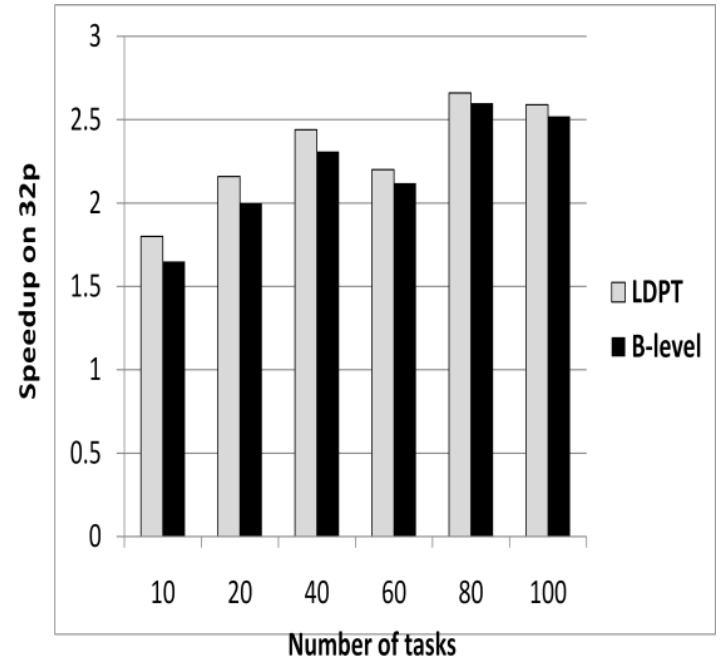

Fig 15: Speedup on 32 processors

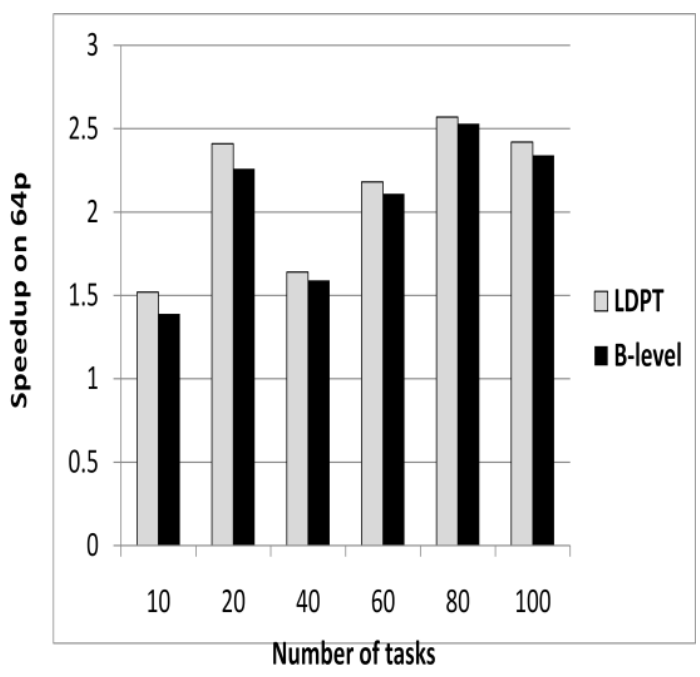

Fig 16: Speedup on 64 processors 


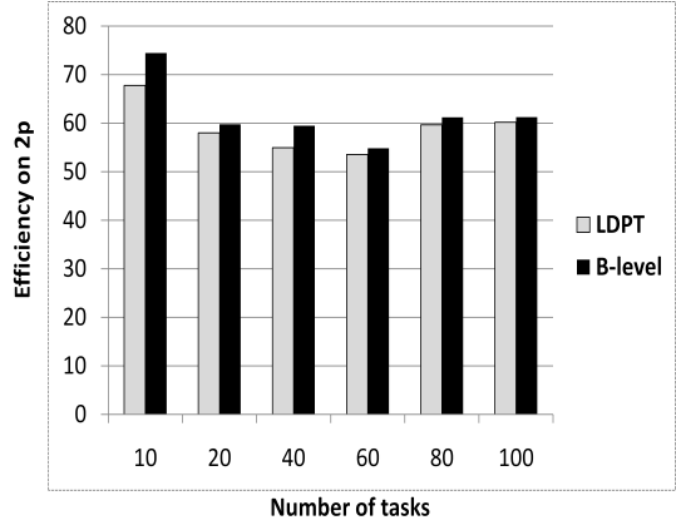

Fig 17: Efficiency on 2 processors

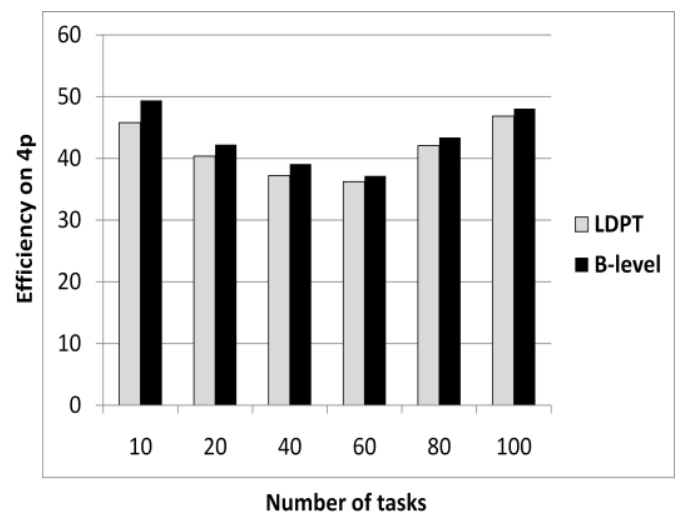

Fig 18: Efficiency on 4 processors

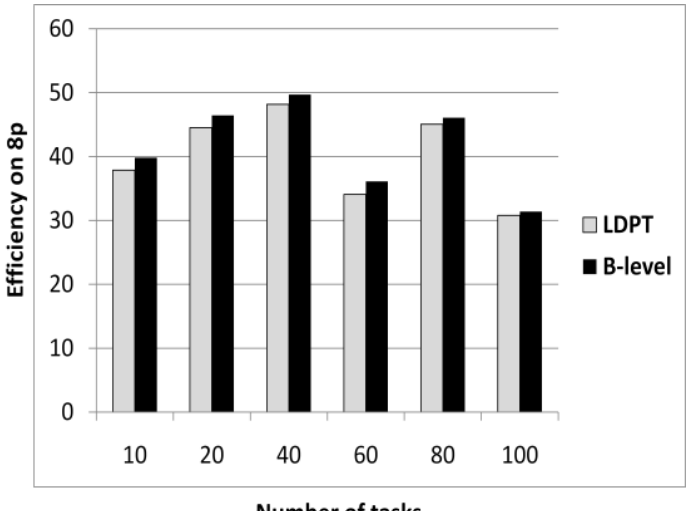

Fig 19: Efficiency on 8 processors

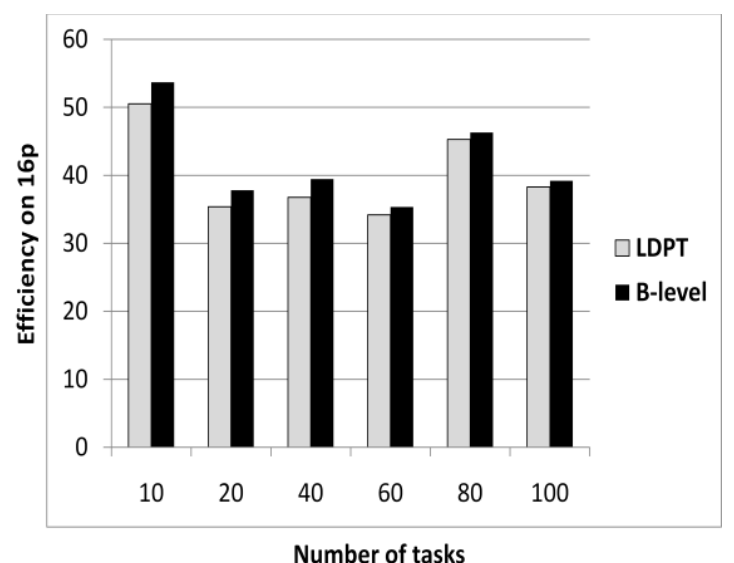

Fig 20: Efficiency on 16 processors

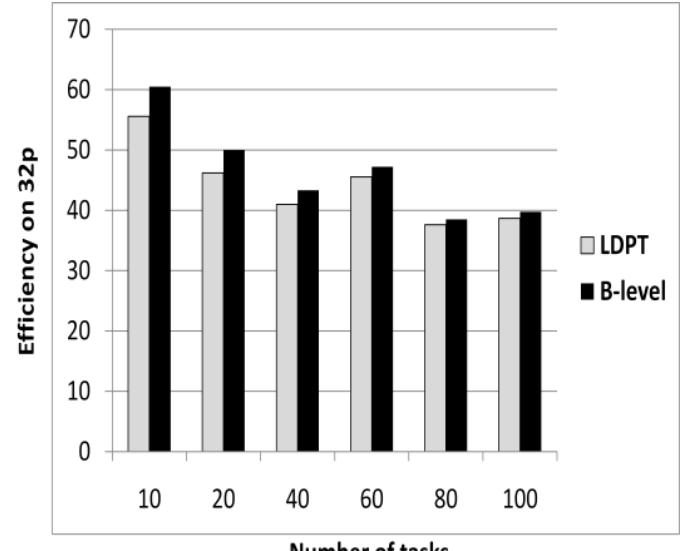

Number of tasks

Fig 21: Efficiency on 32 processors

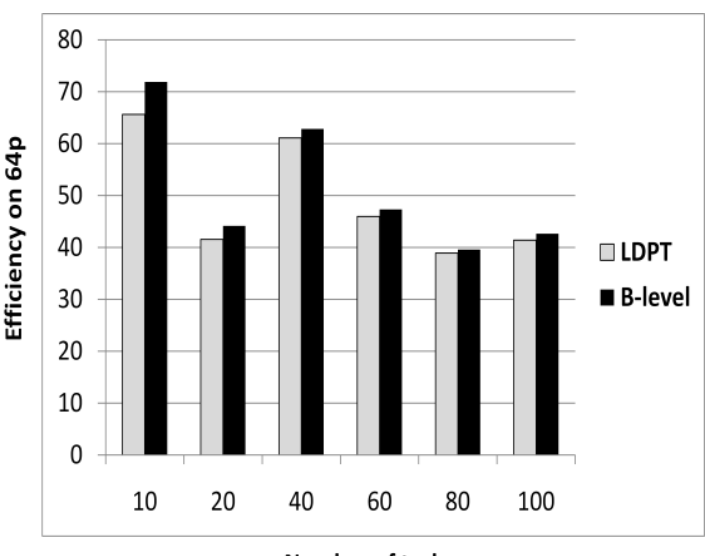

Number of tasks

Fig 22: Efficiency on 64 processors

Figure 17, 18, 19, 20, 21, 22 depict efficiency versus number of processors with varying number of tasks $(20,40,60,80,100)$. It is shown that LDPT algorithm is more efficient and provide better performance than B-level algorithm. Most of processors elements have been perfect utilized in our algorithm because of the communications among tasks is not affected in algorithm breadth procedures.

\section{DISCUSSION OF ENERGY AWARENESS}

EASy (Energy Aware Scheduling) algorithm is an algorithm that aims to reduce power consumption. EASy is divided into two phases. In the first phase B-level algorithm is used for scheduling. In this paper, we have developed a new static scheduling algorithm called LDPT (Leveled DAG Prioritized Task). LDPT outperforms B-level in terms of schedule length, speedup, and efficiency. The second phase of EASy algorithm involves applying a technique called DVFS (Dynamic Voltage Frequency Scaling) [18] for power reduction. We expect good results when applying LDPT instead of B-level with this technique (DVFS).

\section{CONCLUSION AND FUTURE WORK}

In this paper, a new static scheduling algorithm (LDPT) is developed for homogeneous distributed computing systems. The performance of LDPT algorithm is compared with the existing B-level algorithm. LDPT is evaluated for different DAGs and found to be giving better results than B-level algorithm in terms of schedule length, speed up, and efficiency with improving ratio $1 \%, 1.58 \%$, and $1.38 \%$ respectively. The future scope of the idea can be as follows: 
- In this paper LDPT algorithm is applied on Directed Acyclic Graph (DAG). In the future it can be applied on Directed Cyclic Graph (DCG)

- LDPT can be applied on Heterogeneous Distributed Computing Systems (HDCS).

- LDPT can be applied in a dynamic strategy instead of static strategy.

- Finally, duplication technique can be applied with LDPT algorithm to minimize the communication overhead.

\section{REFERENCES}

[1] Journal of Theoretical and Applied Information Technology. (2011, April 9). [Online]. Available: http://www.jatit.org/distributed-computing/grid-vsdistributed.htm.

[2] H. Topcuoglu, S. Hariri, and M.Y. Wu, "PerformanceEffective and Low-Complexity Task Scheduling for Heterogeneous Computing," IEEE Trans. Parallel and Distributed Systems, Vol. 13, No.3, pp. 260-274, March 2002.

[3] Y.K. Kwok and I. Ahmad, "Static Scheduling Algorithms for allocating Directed Task Graphs to Multiprocessors", ACM Computing Surveys, Vol.31, No.4, pp. 406-471, December 1999.

[4] Topcuoglu, H., Hariri, S., Wu, M.Y. "Performance effective and low complexity task scheduling for heterogonous computing", IEEE Trans. Parallel Distributed Syst. 13(3), 2002.

[5] Luiz F. Bittencourt, Rizos Sakellariou. "DAG Scheduling Using a Look ahead Variant of the Heterogeneous Earliest Finish Time Algorithm", 18th Euromicro International Conference on Parallel, Distributed and Network-Based Processing (PDP), pp. 27-34, 2010.

[6] Eswari, R. and Nickolas, S. "Path-Based Heuristic Task Scheduling Algorithm for Heterogeneous Distributed Computing Systems". Advances in Recent Technologies in Communication and Computing (ARTCom), International Conference on 2010. P: 30-34

[7] Rajak and Ranjit. "A Novel Approach for Task Scheduling in Multiprocessor System". International Journal of Computer Applications (IJCA), Vol.44, No. 11, pp. 12-16. April 2012.

[8] Ahmad, S.G.; Munir, E.U. and Nisar, W. PEGA "A Performance Effective Genetic Algorithm for Task
Scheduling in Heterogeneous Systems". High Performance Computing and Communication \& 2012 IEEE 9th International Conference on Embedded Software and Systems (HPCC-ICESS), IEEE 14th International Conference on 2012. Pp. 1082-1087.

[9] Tang, X., et al., "List scheduling with duplication for heterogeneous computing systems", Journal of Parallel and Distributed Computing (JPDC), Vol. 70, No.4, pp. 323-329. 2010.

[10] Nasri,W. and Nafti, W. "A new DAG scheduling algorithm for heterogeneous platforms". Parallel Distributed and Grid Computing (PDGC), second IEEE International Conference on 2012. Pp. 114-119.

[11] B. Kruatrachue and T. Lewis, "Grain size determination for parallel processing," IEEE Software, vol. 5, no. 1, pp. 23-32, May 1988.

[12] M. Y. Wu and D. D. Gajski, "Hypercool: a programming aid for message passing systems," IEEE Transactions on Parallel and Distributed Systems, vol. I, no. 3 pp. 330343, July 1990.

[13] J. J. Hwang. Y.C. Chow. F. D. Anger and C.-Y. Lee. "Scheduling precedence graphs In systems with interprocessor communication times." SLAM Journal of Computing, vol. 18, no. 2. pp. 244-257. 1989.

[14] G.C. Slh and E. A. Lee. "A compile-time scheduling heuristic for interconnection-constrained heterogeneous processor architectures." IEEE Transactions on Parallel and Distributed Systems, vol. 4. no. 2, pp. 75-87. Feb. 1997.

[15] H. El-Rewini and T.G .Lewis, " Scheduling parallel programs onto arbitrary target machines." Journal of Parallel and Distributed Computing, vol. 9. no. 2, pp. 138 153, June 1990.

[16] Panos M. Pardalos, SanguthevarRajasekaran, José D. P. Rolim, " Randomization Methods in Algorithm Design: DIMACS Workshop", vol. 43, December 12-14, 1997.

[17] Ebrahimirad, V.; Rajabi, A.; Goudarzi, M., "Energy-aware scheduling algorithm for precedence-constrained parallel tasks of network-intensive applications in a distributed homogeneous environment". Computer and Knowledge Engineering (ICCKE) 3th International Conference on 2013. Pp. $368-375$.

[18] Zhuravlev, S., et al., Survey of energy-cognizant scheduling techniques. 2012. 